\title{
Parental awareness of food portion sizes for children aged 6-36 months
}

\author{
M. A. Kerr, T. A. McCrorie, J. M. W. Wallace and M. B. E. Livingstone \\ Northern Ireland Centre for Food and Health, University of Ulster, Coleraine, BT52 1SA, UK
}

\begin{abstract}
A key stage for establishing healthy eating patterns is in the first few years of life, particularly from birth to 36 months (m) ${ }^{(1-4)}$. Despite the potential link between portion size and over eating, quantitative national dietary guidance for this age group is lacking. This study therefore aimed to investigate the attitudes and experiences of parents when determining food portion sizes for young children aged 6-36 m.

An online survey was created using a web-based survey builder (Survey Monkey ${ }^{\circledR}$ ). Respondents were parents/carers of at least one child aged 6-36 m. Question topics included demographics, portion control techniques and common portion size information sources. Parents/carers were asked to take part in the study via email distribution lists from a mother and baby club within a leading baby feeding company ${ }^{(5)}$.

A total of 917 parents/carers took part in the survey, with $90.7 \%$ of respondents completing all questions $(n$ 832). The majority of respondents were aged 28-32 y $(n$ 295; 32.2\%), from England $(n 784 ; 85.5 \%)$, urban dwelling $(n$ 539; 58.8\%) and employed $(n$ 557; $60.6 \%$ ). A total of $35.2 \%$ of respondents felt that not enough information on portion size was currently available compared to $15.6 \%$ who were satisfied with the current information available. A large proportion of respondents included using their own judgement to determine an appropriate portion size for their infant/toddler $(95.8 \%)$. Only $20.9 \%$ of respondents reported seeking portion size advice from a healthcare professional (Table 1).
\end{abstract}

\begin{tabular}{lcc}
\hline $\begin{array}{l}\text { Where do you normally seek advice for } \\
\text { appropriate portion sizes for your child }\end{array}$ & & \\
aged 6-36 months? & $n$ & $\mathbf{9 5 . 8}$ \\
\hline Use my own judgement/experience & 802 & $\mathbf{3 1 . 1}$ \\
Family and friends & 260 & $\mathbf{2 3 . 7}$ \\
Side of packet/jar & 198 & $\mathbf{2 0 . 9}$ \\
Guidance from health professionals & 175 & $\mathbf{2 0 . 1}$ \\
Recipe books & 168 & $\mathbf{5 . 7}$ \\
Websites & 48 & $\mathbf{1 . 3}$ \\
TV & 11 & \\
\hline
\end{tabular}

This research suggests that many parents feel they do not currently have access to sufficient information on appropriate food portion sizes for their infants and toddlers aged 6-36 $\mathrm{m}$. These results highlight a need to compare parental perceptions of appropriate food portion sizes with existing guidance for this age group. Investigation of the use of training resources by healthcare professionals for disseminating infant/toddler portion size information may also be warranted.

This research was funded by Danone Baby Nutrition, UK (Nutricia Ltd). Ethical approval was obtained from the Biomedical Sciences Ethics filter committee at the University of Ulster.

1. Birch LL \& Fisher JO (1998) Pediatrics 101, 539-549.

2. Birch LL (1999) Annu Rev Nutr 19, 41-62.

3. Skinner JD, Carruth BR, Bounds W, et al. (2002) JAMA 102, 1638-1647.

4. Coulthard H, Harris G \& Emmett P (2010) Public Health Nutrition 13, 2044-2051.

5. Cow and Gate Mum \& Baby Club: http://www.cowandgate.co.uk/register?tr=loh. Accessed 26.03.12. 